\title{
On a linearly damped 2 body problem
}

\author{
Alain Haraux \\ Sorbonne Université, Université Paris-Diderot SPC, CNRS, INRIA, \\ Laboratoire Jacques-Louis Lions, LJLL, F-75005, Paris, France. \\ e-mail: haraux@ann.jussieu.fr
}




\begin{abstract}
The usual equation for both motions of a single planet around the sun and electrons in the deterministic Rutherford-Bohr atomic model is conservative with a singular potential at the origin. When a dissipation is added, new phenomena appear. It is shown that whenever the momentum is not zero, the moving particle does not reach the center in finite time and its displacement does not blow-up either, even in the classical context where arbitrarily large velocities are allowed. Moreover we prove that all bounded solutions tend to 0 for $t$ large, and some formal calculations suggest the existence of special orbits with an asymptotically spiraling exponentially fast convergence to the center.
\end{abstract}

Key words: gravitation, singular potential, global solutions, spiraling orbit. 


\section{Introduction}

The usual equation for both motions of a single planet around the sun and electrons in the deterministic Rutherford-Bohr atomic model is conservative with a singular potential at the origin. In the previous paper [3], the author raised the hypothesis that some phenomena such as carboniferous gigantism of arthropods and vegetals (cf.[2, 5]), the appearent expansion of the universe (cf.[4])or slight deformation of electrical devices over decades might be explained by an atomic contraction phenomenon (with respect to the size of the proton) coming from a very weak environmental dissipation produced by what we consider to be vacuum. When a dissipation is added to the basic equation

$$
m u^{\prime \prime}=-\frac{q^{2}}{4 \pi \varepsilon_{0}} \frac{u}{|u|^{3}}
$$

modelling Coulomb's central force (with $q$ the elementary charge, $\mathrm{m}$ the mass of the electron and $\varepsilon_{0}$ the vacuum permittivity) or its equivalent Newton's law for planets (where $G$ is the gravitational constant and $M_{S}$ the mass of the sun)

$$
u^{\prime \prime}=-G M_{S} \frac{u}{|u|^{3}}
$$

written in complex form in the orbital plane with a suitable set of axes and length unit, new interesting phenomena appear. This leads us to consider, as the simplest possible dissipative perturbation of the conservative problem

$$
u^{\prime \prime}+c_{0} \frac{u}{|u|^{3}}=0
$$

including both equations, the modified ODE

$$
u^{\prime \prime}+\delta u^{\prime}+c_{0} \frac{u}{|u|^{3}}=0
$$

In [3], considering the difficulty of exhibiting decaying solutions for the equation (2), the author investigated a slightly more complicated case where we allow the charge $q$ to decay exponentially in time. This led to the equation

$$
u^{\prime \prime}+\delta u^{\prime}+c_{0} e^{-\alpha t} \frac{u}{|u|^{3}}=0
$$

for which explicit exponentially decaying solutions depending on two real parameters can be exhibited. More precisely for $\alpha=\delta$ we found the fast rotating spiraling solutions

$$
u_{ \pm}(t)=U e^{-\delta t} \exp \left( \pm i \frac{\sqrt{c_{0}}}{\delta U^{3 / 2}} e^{\delta t}+i \phi\right)
$$

whereas for $\alpha=\frac{3}{2} \delta$, we found the uniformly rotating spiraling solutions

$$
v_{ \pm}(t)=V e^{-\frac{\delta}{2} t} \exp \left( \pm i t \sqrt{V^{-3} c_{0}+\frac{\delta^{2}}{4}}+i \phi\right)
$$

Although it would probably be interesting to do, at the level of [3] we did not try to prove wellposedness (global existence) for general initial data not leading to collision with the center, nor 
did we try to elucidate global behavior of trajectories other than the previous special solutions. Before deciding to do that, one must first try to see whether or not the simpler model (2) is sufficient to identify a contraction phenomenon, and possibly determine the extent of its stability.

In the present paper, we derive some partial results on (2) together with some heuristic discussion. More precisely, in Section 2, we prove that whenever the momentum is not zero, the moving particle does not reach the center in finite time and its displacement does not blow-up in finite time either, even in the classical context where arbitrarily large velocities are allowed. In Section 3, we prove that $u(t)$ remains bounded under a smallness condition involving both initial radius and velocity. In Section 4, we prove that all bounded non-vanishing solutions converge to 0 at infinity in $t$. Finally, in Section 5, some formal calculations suggest the existence of special orbits with an asymptotically spiraling convergence to the center. We conclude by a few heuristic remarks.

\section{A global existence result}

For convenience we recall our basic equation (2)

$$
u^{\prime \prime}+\delta u^{\prime}+c \frac{u}{|u|^{3}}=0
$$

where we simply wrote $c$ instead of $c_{0}$, since for mathematicians $c$ is not always the velocity of light. In order to solve this equation with $u=u(t) \in \mathbb{R}^{2}=\mathbb{C}$ we introduce the amplitude and the phase

$$
u(t)=r(t) e^{i \theta(t)}
$$

and we shall drop the variable $t$ when it does not lead to confusion. Here all derivatives are with respect to $t$. From the formulas

$$
\begin{gathered}
u^{\prime}=r^{\prime} e^{i \theta}+i r \theta^{\prime} e^{i \theta}=\left(r^{\prime}+i r \theta^{\prime}\right) e^{i \theta}=\left(r^{\prime}+i r \theta^{\prime}\right) \frac{u}{|u|} \\
u^{\prime \prime}=\left[\left(r^{\prime \prime}-r \theta^{\prime 2}\right)+i\left(2 r^{\prime} \theta^{\prime}+r \theta^{\prime \prime}\right)\right] \frac{u}{|u|}
\end{gathered}
$$

we conclude that (4) is equivalent to the system of two real equations

$$
\begin{gathered}
r^{\prime \prime}-r \theta^{\prime 2}+\delta r^{\prime}+\frac{c}{r^{2}}=0 \\
2 r^{\prime} \theta^{\prime}+r \theta^{\prime \prime}+\delta r \theta^{\prime}=0
\end{gathered}
$$

We observe first that when trying to solve (4) for $t \geq 0$, the initial value $u(0)=0$ is excluded by the singularity, while whenever $u(0) \neq 0$ we shall obtain at least a local solution for any initial velocity $u^{\prime}(0)$. Moreover, multiplying (6) by $r$ we reduce it to

$$
\left(r^{2} \theta^{\prime}\right)^{\prime}+\delta r^{2} \theta^{\prime}=0
$$

so that

$$
\forall t \geq 0, \quad r^{2}(t) \theta^{\prime}(t)=M e^{-\delta t}
$$


with

$$
M=: r^{2}(0) \theta^{\prime}(0)
$$

This relation expresses the variation of the momentum of the solution, which was constant in the conservative case $\delta=0$ and decays exponentially when $\delta>0$. By plugging in (5) the value of $\theta^{\prime}$ given by (7), we are left with an equation involving $r(t)$ only:

$$
r^{\prime \prime}-\frac{M^{2} e^{-2 \delta t}}{r^{3}}+\frac{c}{r^{2}}+\delta r^{\prime}=0
$$

We now state our first result

Theorem 2.1. Assuming $M \neq 0$, the unique local non-vanishing solution $r=r(t)$ of $(8)$ with any initial data $r(0)=r_{0} \neq 0 ; r^{\prime}(0)=r_{0}^{\prime} \in \mathbb{R}$ is global and we have for some constants $C>0, \eta>0$

$$
\begin{array}{ll}
\forall t \geq 0, & \left|r^{\prime}(t)\right| \leq C e^{\delta t} \\
\forall t \geq 0, & r(t) \geq \eta e^{-2 \delta t}
\end{array}
$$

Proof. The existence and uniqueness of local non-vanishing solutions is obvious. Let us introduce the scalar function $F$, defined as long as the solution $r$ exists and does not vanish by the formula:

$$
F(t):=\frac{r^{\prime 2}(t)}{2}-\frac{c}{r(t)}+\frac{M^{2}}{2} \frac{e^{-2 \delta t}}{r^{2}(t)}
$$

We have immediately

$$
F^{\prime}(t)=r^{\prime}(t)\left(r^{\prime \prime}(t)+\frac{c}{r^{2}(t)}-\frac{M^{2} e^{-2 \delta t}}{r^{3}(t)}\right)-\frac{M^{2} \delta e^{-2 \delta t}}{r^{2}(t)}=-\delta r^{\prime 2}(t)-\frac{M^{2} \delta e^{-2 \delta t}}{r^{2}(t)}<0
$$

In particular for all $t \in[0, T \max )$ we have

$$
\frac{r^{\prime 2}(t)}{2}-\frac{c}{r(t)}+\frac{M^{2}}{2} \frac{e^{-2 \delta t}}{r^{2}(t)} \leq F_{0}:=F(0)
$$

which can be rearranged in the more convenient form

$$
\frac{r^{\prime 2}(t)}{2}+\frac{M^{2}}{2}\left(\frac{e^{-\delta t}}{r(t)}-\frac{c}{M^{2}} e^{\delta t}\right)^{2} \leq F_{0}+\frac{c^{2}}{2 M^{2}} e^{2 \delta t}
$$

providing at once the two inequalities

$$
\forall t \in[0, T \max ), \quad r^{\prime 2}(t) \leq 2\left(F_{0}+\frac{c^{2}}{2 M^{2}} e^{2 \delta t}\right) \leq\left(2 F_{0}+\frac{c^{2}}{M^{2}}\right) e^{2 \delta t}
$$

and

$$
\forall t \in[0, T \max ), \quad \frac{e^{-\delta t}}{r(t)} \leq \frac{c}{M^{2}} e^{\delta t}+\sqrt{\frac{2}{M^{2}} F_{0}+\frac{c^{2}}{M^{4}} e^{2 \delta t}} \leq K e^{\delta t}
$$

The conclusions follow immediately with

$$
C=\left(2 F_{0}+\frac{c^{2}}{M^{2}}\right)^{1 / 2} ; \quad \eta=\frac{1}{K} .
$$


Corollary 2.2. Assume

$$
\operatorname{Im}\left(\overline{u_{0}} u_{0}^{\prime}\right) \neq 0
$$

Then the local solution of (4) with initial conditions $u(0)=u_{0} ; u^{\prime}(0)=u_{0}^{\prime}$ is global and satisfies for some constants $D>0, \eta>0$

$$
\begin{aligned}
& \forall t \geq 0, \quad\left|u^{\prime}(t)\right| \leq D e^{\delta t} \\
& \forall t \geq 0, \quad|u(t)| \geq \eta e^{-2 \delta t}
\end{aligned}
$$

Proof. I suffices to prove that the two above inequalities are satisfied for all $t \in[0, T \max )$, the maximal existence time for a non-vanishing solution $u$ of (4) with initial conditions $u(0)=$ $u_{0} ; u^{\prime}(0)=u_{0}^{\prime}$. Selecting for $\theta$ a continuous determination of the argument of $u(t)$, the pair $(r, \theta)$ satisfies the equivalent system of equations (5)-(6). In addition an immediate calculation shows that $\theta^{\prime}=\operatorname{Im}\left(\frac{\bar{u}}{|u|} u^{\prime}\right)$ and in particular

$$
\operatorname{Im}\left(\overline{u_{0}} u_{0}^{\prime}\right)=r(0) \theta^{\prime}(0)
$$

By the previous theorem, the solution $r(t)$ of the scalar equation for the radius is global and satisfies the concluding inequalities. Then the pair $(r, \theta)$ correspond to the non-vanishing solution $u$ of (4) with initial conditions $u(0)=u_{0} ; u^{\prime}(0)=u_{0}^{\prime}$ which is therefore global with $|u(t)| \geq \eta e^{-2 \delta t}$. For the last inequality we observe that

$$
\mid \theta^{\prime}(t) r(t) \leq M e^{-\delta t} r^{-1}(t) \leq \frac{M}{\eta} e^{\delta t}
$$

Then

$$
\left|u^{\prime}(t)\right|=\left|r^{\prime}+i r \theta^{\prime}\right| \leq\left|r^{\prime}(t)\right|+\left|r(t) \theta^{\prime}(t)\right| \leq\left(C+\frac{M}{\eta}\right) e^{\delta t}:=D e^{\delta t}
$$

\section{$3 \quad$ Bounded trajectories}

The next result does not require the condition $M \neq 0$.

Theorem 3.1. Let $u_{0} \neq 0$ and assume the initial smallness condition

$$
\left|u_{0}\right|\left|u_{0}^{\prime}\right|^{2}<2 c
$$

Then the local solution $u$ of $(4)$ on $[0, T)$ with initial conditions $u(0)=u_{0} ; u^{\prime}(0)=u_{0}^{\prime}$ satisfies the inequality

$$
\forall t \in[0, T), \quad|u(t)| \leq \frac{2 c\left|u_{0}\right|}{2 c-\left|u_{0}\right|\left|u_{0}^{\prime}\right|^{2}}
$$

In particular if $u$ does not vanish in finite time, $u$ is a global bounded non-vanishing solution .

Proof. We start from the inequality

$$
\frac{r^{\prime 2}(t)}{2}-\frac{c}{r(t)}+\frac{M^{2}}{2} \frac{e^{-2 \delta t}}{r^{2}(t)} \leq F_{0}=\frac{r_{0}^{\prime 2}}{2}-\frac{c}{r_{0}}+\frac{M^{2}}{2 r_{0}^{2}}
$$


and we observe that if $F_{0}<0$, the inequality $\frac{c}{r(t)} \geq-F_{0}=\left|F_{0}\right|$ implies $r(t) \leq \frac{c}{\left|F_{0}\right|}$. Now we compute $F_{0}$ in terms of the initial data. In fact we have the formula

$$
\left|u^{\prime}\right|^{2}=r^{\prime 2}+\theta^{\prime 2} r^{2}=r^{\prime 2}+\frac{M^{2}}{r^{2}}
$$

so that for $t=0$ we find

$$
F_{0}=\frac{\left|u_{0}^{\prime}\right|^{2}}{2}-\frac{c}{r_{0}}=\frac{\left|u_{0}^{\prime}\right|^{2}}{2}-\frac{c}{\left|u_{0}\right|}
$$

therefore the condition $F_{0}<0$ is equivalent to $\left|u_{0}\right|\left|u_{0}^{\prime}\right|^{2}<2 c$. The previous observation now gives that under condition (14), we have

$$
r(t) \leq \frac{c}{\frac{c}{\left|u_{0}\right|}-\frac{\left|u_{0}^{\prime}\right|^{2}}{2}}=\frac{2 c\left|u_{0}\right|}{2 c-\left|u_{0}\right|\left|u_{0}^{\prime}\right|^{2}}
$$

\section{Convergence to 0 of non-vanishing bounded solutions}

As in the case of the conservative equation (1) which is well known to have elliptic and parabolic trajectories, we suspect that (4) may have some unbounded solutions. But the next result shows that in sharp constrast with (1), (4) does not have any periodic trajectory at all.

Theorem 4.1. For any solution $u$ of (4) such as $|u(t)|$ is global, positive and bounded on $\mathbb{R}^{+}$, we have

$$
\lim _{t \rightarrow+\infty}|u(t)|=0
$$

Proof. We introduce the total energy

$$
E(t):=\frac{1}{2}\left|u^{\prime}\right|^{2}(t)-\frac{c}{|u(t)|}
$$

Since $u$ never vanishes, it is clear that $u \in C^{2}\left(\mathbb{R}^{+}\right)$and we have

$$
E^{\prime}(t)=-\delta\left|u^{\prime}(t)\right|^{2}
$$

In particular, $E(t)$ is non-increasing. Then we have two possibilities

\section{Case 1}

$$
\lim _{t \rightarrow+\infty} E(t)=-\infty
$$

Then since $\frac{c}{|u(t)|} \geq-E(t)$ we conclude that

$$
\lim _{t \rightarrow+\infty}|u(t)|=0
$$




\section{Case 2}

$$
\lim _{t \rightarrow+\infty} E(t)=E^{*}>-\infty
$$

Then

$$
\forall t \in \mathbb{R}^{+}, \quad E(0)-E(t)=\delta \int_{0}^{t} \mid u^{\prime}\left(\left.s\right|^{2} d s\right.
$$

In particular $u^{\prime} \in L^{2}\left(\mathbb{R}^{+}, \mathbb{C}\right)$. We have assumed that $u(t)$ is bounded, hence precompact in $\mathbb{R}^{+}$ with values in $\mathbb{C}$. Therefore if (16) is not satisfied we may assume that for some sequence $t_{n}$ tending to $+\infty$

$$
\lim _{n \rightarrow+\infty} u\left(t_{n}\right)=w \neq 0
$$

On the other hand we have

$$
\lim _{n \rightarrow+\infty} u^{\prime}\left(t_{n}+s\right)=0
$$

in the strong topology of $L^{2}(0,1)$ and in particular, by Cauchy-Schwarz-inequality we find

$$
\lim _{n \rightarrow+\infty} u\left(t_{n}+s\right)=w
$$

uniformly on $[0,1]$. Since $w \neq 0$ this implies

$$
\lim _{n \rightarrow+\infty} c \frac{u\left(t_{n}+s\right)}{\left|u\left(t_{n}+s\right)\right|^{2}}=c \frac{w}{|w|^{2}}
$$

uniformly on $[0,1]$. But then by the equation

$$
\lim _{n \rightarrow+\infty} u^{\prime \prime}\left(t_{n}+s\right)=c \frac{w}{|w|^{2}}
$$

in the strong topology of $L^{2}(0,1)$. This is contradictory with (21). Indeed it implies for instance

$$
\lim _{n \rightarrow+\infty} \int_{0}^{1} s(1-s) u^{\prime \prime}\left(t_{n}+s\right) d s=c \int_{0}^{1} s(1-s) d s \frac{w}{|w|^{2}}=z \neq 0
$$

while on the other hand

$$
\int_{0}^{1} s(1-s) u^{\prime \prime}\left(t_{n}+s\right) d s=-\int_{0}^{1}(1-2 s) u^{\prime}\left(t_{n}+s\right) d s \longrightarrow 0
$$

This contradiction concludes the proof.

As an immediate consequence of the previous theorem and the results of Sections 2 and 3, we obtain

Corollary 4.2. Assume $\operatorname{Im}\left(\overline{u_{0}} u_{0}^{\prime}\right) \neq 0$ and $\left|u_{0}\right|\left|u_{0}^{\prime}\right|^{2}<2 c$. Then the local solution of (4) with initial conditions $u(0)=u_{0} ; u^{\prime}(0)=u_{0}^{\prime}$ tends to 0 as $t$ tends to infinity. 


\section{A formal calculation for spiraling solutions}

The previous theorem, proved by contradiction, is not constructive and therefore does not provide any hint on the rate of decay to 0 of bounded solutions. In the previous paper [3] we could not identify any exponentially decaying solution of (2). We now make a formal calculation suggesting that such solutions might exist after all. The starting idea is to imitate the circular trajectories of the conservative problem, observing that such trajectories correspond to a constant angular velocity and for them the nonlinear term of the radial equation vanishes. When we try to do that for the damped equation we find

$$
r(t)=\frac{M^{2}}{c} e^{-2 \delta t}
$$

This is not a true solution of the equation since then

$$
r^{\prime \prime}+\delta r^{\prime}=\frac{M^{2}}{c}\left(4 \delta^{2}-2 \delta\right) e^{-2 \delta t} \neq 0
$$

but at least the result is non-singular and even becomes very small if $\delta$ is small. This gives the idea to look for a solution of the form

$$
r(t)=\frac{M^{2}}{c} e^{-2 \delta t}+\varepsilon(t)
$$

where $\varepsilon(t)$ would be negligible with respect to $\frac{M^{2}}{c} e^{-2 \delta t}$, either uniformly, or at least for $t$ large. Plugging this in the equation we find

$$
c \varepsilon(t)=-r^{3}\left(r^{\prime \prime}+\delta r^{\prime}\right)
$$

If now we replace both

$$
r^{3} \sim \frac{M^{6}}{c^{3}} e^{-6 \delta t} ; \quad r^{\prime \prime}+\delta r^{\prime} \sim \frac{M^{2}}{c}\left(4 \delta^{2}-2 \delta\right) e^{-2 t}
$$

by their alleged "main part" we find

$$
\varepsilon(t) \sim \frac{M^{8}}{c^{5}}\left(2 \delta-4 \delta^{2}\right) e^{-8 \delta t}
$$

which is indeed quite negligible with respect to $\frac{M^{2}}{c} e^{-2 \delta t}$ for $t$ large and even uniformly if $\delta$ and $\frac{M^{6}}{c^{4}}$ are both small enough. To see the rule we main consider trying the next step. the calculations are slightly more involved but we get rather easily

$$
\varepsilon(t) \sim \frac{M^{8}}{c^{5}}\left(2 \delta-4 \delta^{2}\right) e^{-8 \delta t}+\frac{M^{14}}{c^{9}}\left(14 \delta-76 \delta^{2}\right)\left(2 \delta-4 \delta^{2}\right) e^{-14 \delta t}
$$

From the algebraic point of view, it is not difficult to see that the process can be continued indefinitely with the appearance of a single new exponential term at each step, deduced from the previous one on multiplication by $\frac{M^{6}}{c^{4}} e^{-6 \delta t}$ times a function of $\delta$ only. So that we can dream of a solution of the form

$$
r(t)=\frac{M^{2}}{c} \sum_{n=0}^{\infty} r_{n}(\delta)\left(\frac{M^{6}}{c^{4}}\right)^{n} e^{-(2+6 n) \delta t}
$$


The only difficulty for convergence will be to control the growth of $r_{n}(\delta)$. At first sight it seems difficult, thinking about the algebra involved, to imagine that this growth is sub-geometric in any range of smallness of $\delta$, a condition which looks almost mandatory for the series to be at least normally convergent for $t$ large. Then in which sense would the above calculations approach a solution? At each step we have an approximate solution with a very close accuracy. And at each step, when we truncate, the behavior is what we expected. For the time being, since the solutions should reasonably be analytic in $t$, the moral of the story remains a mystery for the author.

\section{Concluding remarks}

The previous calculations suggest the possible existence of special orbits with an asymptotically (exponentially fast) spiraling convergence to the center. More precisely if we succeed in finding, by whichever method, a solution $r$ of (8)with

$$
r(t) \sim C e^{-2 \delta t}
$$

for a certain $C=C(M)>0$, from this we can build a solution $u=r e^{i \theta}$ with

$$
\theta^{\prime 2} \sim K e^{6 \delta t}
$$

so that $\left|u^{\prime}\right|^{2}=r^{\prime 2}+\theta^{\prime 2} r^{2} \geq k e^{2 \delta t}$ for some positive $k$ and $t$ large. The kinetic energy blows-up exponentially in such a case, which is not absurd since the potential energy also.

Remark 6.1. This construction seems interesting, but even in case it works this is not entirely satisfactory for the following reason: as in the case of (3), the family of spiraling orbits built in this way would depend on only two real parameters (the moment and the initial phase) whereas the phase space has four dimensions.

Remark 6.2. For the moment, despite the formal calculation of the previous section, we know nothing about the rate of decay of any solution to 0 . The only certain information that we have on asymptotics is the inequality $r(t) \geq \eta e^{-2 \delta t}$ for some $\eta>0$. This inequality can be refined asymptotically, relying on the inequality

to yield

$$
\forall t \in[0, T \max ), \quad \frac{e^{-\delta t}}{r(t)} \leq \frac{c}{M^{2}} e^{\delta t}+\sqrt{\frac{2}{M^{2}} F_{0}+\frac{c^{2}}{M^{4}} e^{2 \delta t}}
$$

$$
\varliminf_{t \rightarrow \infty} r(t) e^{2 \delta t} \geq \frac{M^{2}}{2 c}
$$

Remark 6.3. In the case $M=0$, it should be possible to study if, as in the conservative case, solutions with initial velocity directed towards the center vanish in finite time, if necessary under some additional conditions. We did not study this question here since it is not our main concern.

Remark 6.4. After the simple model of Rutherford-Bohr explained in [1, 6] and after the introduction of undulatory mechanics by L. De Broglie, the purely probabilistic model of E. Schrodinger (cf. [7]) has been considered the best atomic model since 1926. It does not seem obvious at all to introduce a damping mechanism in that model, and it might very well happen that in order to do that, an effective coupling between deterministic corpuscular and probabilistic wave conceptions of particles has to be devised, as was always advocated by L. De Broglie and even suggested by E. Schrodinger himself in [7]. 


\section{References}

[1] N.Bohr, On the Constitution of Atoms and Molecules, Philosophical Magazine, Series 26, vol. 1, (July 1913) , p.1-24.

[2] J.F.Harrison, A.Kaiser and J.M. VandenBrooks ; Atmospheric oxygen level and the evolution of insect body size. Proceedings of the Royal Society B. 277(2010):1937-1946. doi:10.1098/rspb.2010.0001

[3] A. Haraux, On Carboniferous Gigantism and Atomic Shrinking. Preprints 2020, 2020110544 (doi: 10.20944/preprints202011.0544.v2).

[4] E.Hubble, M.L. Humason, The Velocity-Distance Relation among Extra-Galactic Nebulae, Astrophysical Journal, vol. 74, p. 43.

[5] Ryssa Parks; An Overview of Hypotheses and Supporting Evidence Regarding Drivers of Insect Gigantism in the Permo-Carboniferous, Western Washington University, Spring 2020 .

[6] E. Rutherford; The Scattering of $\alpha$ and $\beta$ Particles by Matter and the Structure of the Atom, E. Rutherford, F.R.S.* Philosophical Magazine, Series 6, vol. 21 May 1911, p. 669-688

[7] E. Schrodinger; An Undulatory Theory of the Mechanics of Atoms and Molecules, Phys. Rev., vol. 28, no 6, decembre 1926, p. 1049-1070 (DOI 10.1103/PhysRev.28.1049) 